\title{
AGR-5/6/7 Final Fuel Characterization
}

\author{
Douglas W. Marshall
}

May 2018

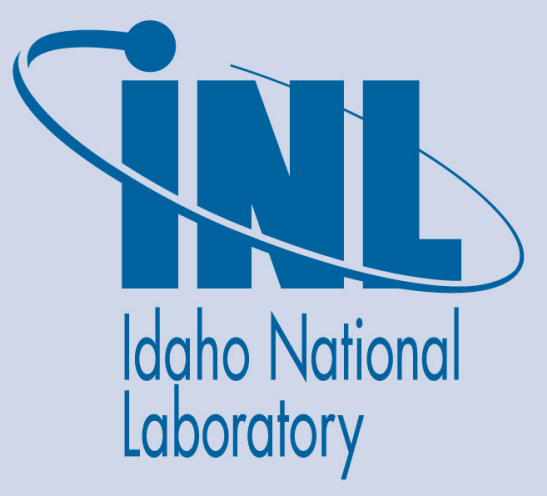

The INL is a U.S. Department of Energy National Laboratory operated by Battelle Energy Alliance 


\title{
AGR-5/6/7 Final Fuel Characterization
}

\author{
Douglas W. Marshall
}

May 2018

\section{Idaho National Laboratory Idaho Falls, Idaho 83415}

http://www.inl.gov

\author{
Prepared for the \\ U.S. Department of Energy \\ Under DOE Idaho Operations Office \\ Contract DE-AC07-05ID14517
}




\section{AGR-5/6/7 'Final' Fuel Characterization}

Douglas Marshall

TRISO Fuel Fabrication Technical Lead

Gas-Cooled Reactor Program Review Meeting May 8, 2018, at Idaho National Laboratory

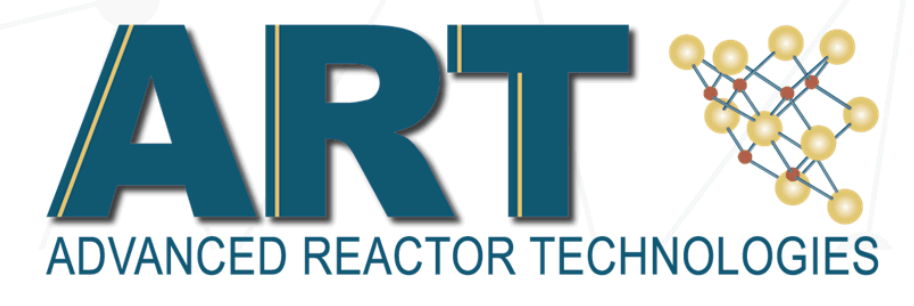




\section{Contents}

- Historical Overview of AGR-5/6/7 Fuel Fabrication

- Kernel Lot

- TRISO Particle Lot

- Compacts Batches

- Kernel Lot Characterization

- TRISO Particle Lot Characterization

- Compact Characterization with Confirmatory Analyses

- 25\% PF Compacts

- 40\% PF Compacts and Overcoated TRISO

- Summary 


\section{Historical Overview - Kernels}

- Formed and sintered in production-scale equipment since AGR-1

- Internal gelation sol-gel process is used

- Chilled uranyl nitrate broth with HMTA, urea, and carbon black dribbled into a column of warm TCE

- Gels are "aged," washed, dried in a collection pot

" Dried (or "green") gels are calcined and sintered in a spouted bed furnace

- Stochastic fissuring potential

- J52L-16-69316 (2013) was too fissured for use

- J52R-16-69317 (2016) had far fewer fissures

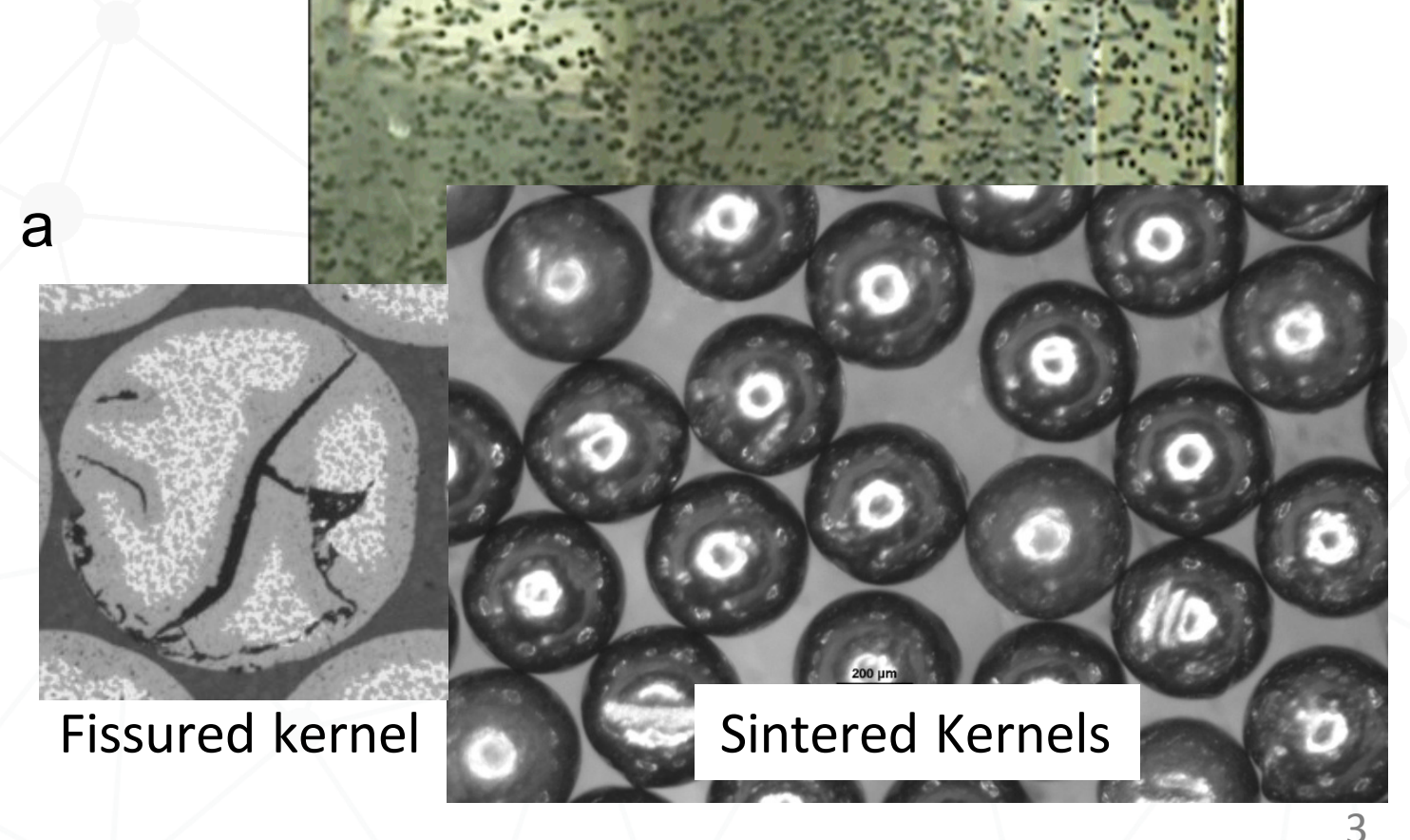




\section{Historical Overview - Kernels (cont.)}

- Composited from 6 kernel sintering batches; yielding $17 \mathrm{~kg}$ of certified kernels in J52R-16-69317

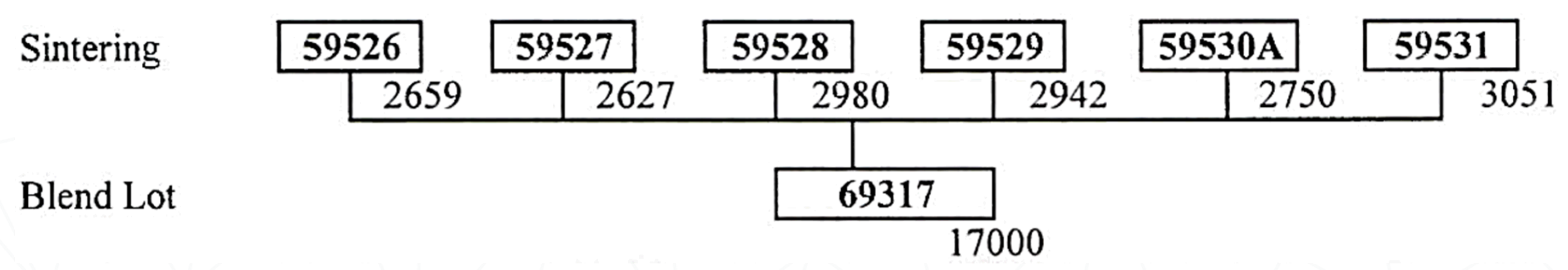




\section{Historical Overview - TRISO}

- Tristructural isotropic (TRISO) fuel particles coated in a spouted bed furnace retort

- AGR-1 and AGR-3/4 coating applied at ORNL in a 2" diameter retort

- AGR-2 and AGR-5/6/7 coated in a small, productionscale (6" diameter) retort

- Four coatings applied in a semi-continuous operation

- Buffer

- Inner pyrocarbon

- Silicon carbide

- Outer pyrocarbon

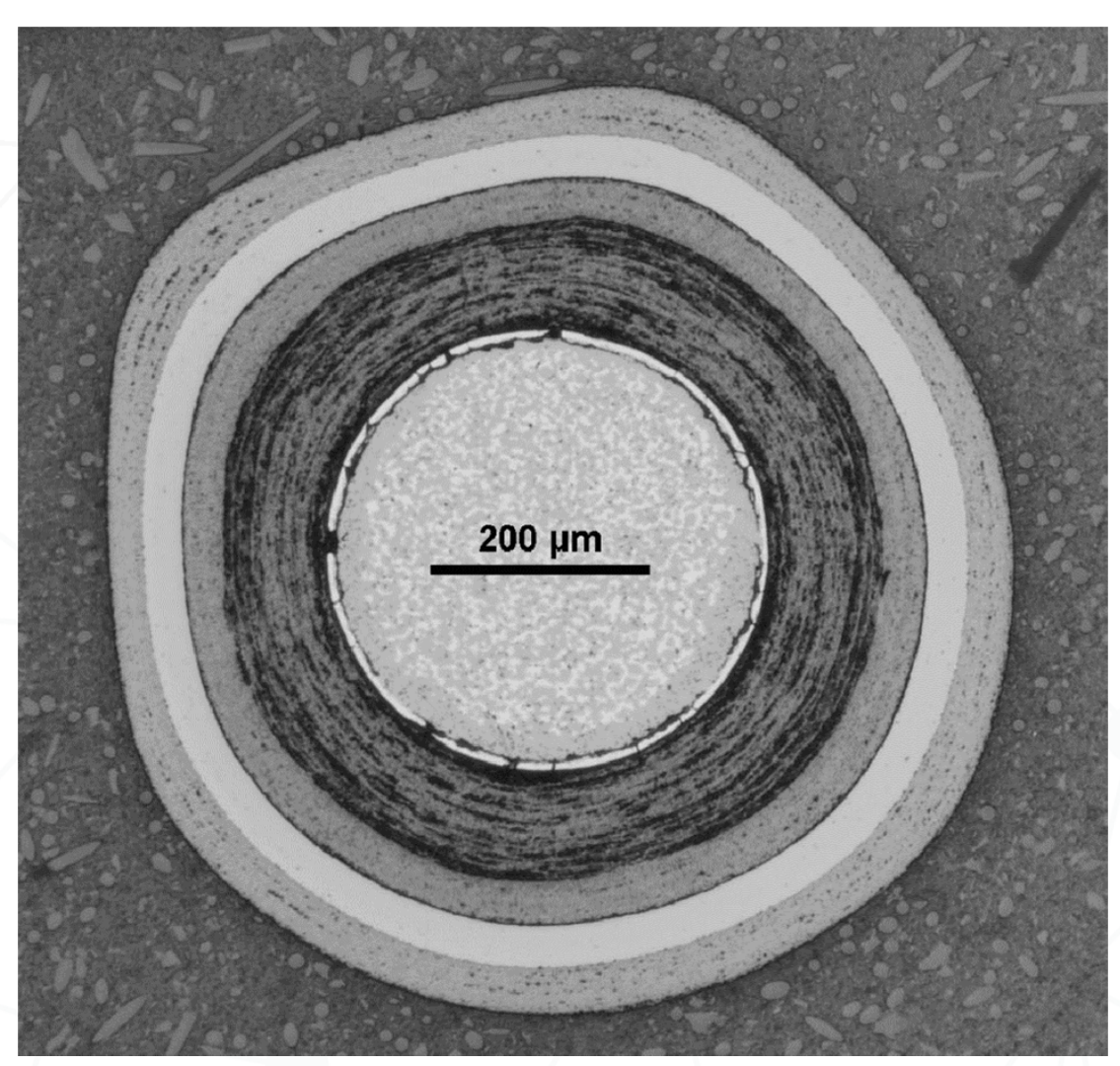

- TRISO particle lot J52R-16-98005 was certified for AGR-5/6/7 in May, 2017 


\section{Historical Overview - TRISO (cont.)}

- Four TRISO batches were composited, after two sieving operations to reject fractured kernels, into a $11.1 \mathrm{~kg}$ TRISO particle lot J52R-16-98005

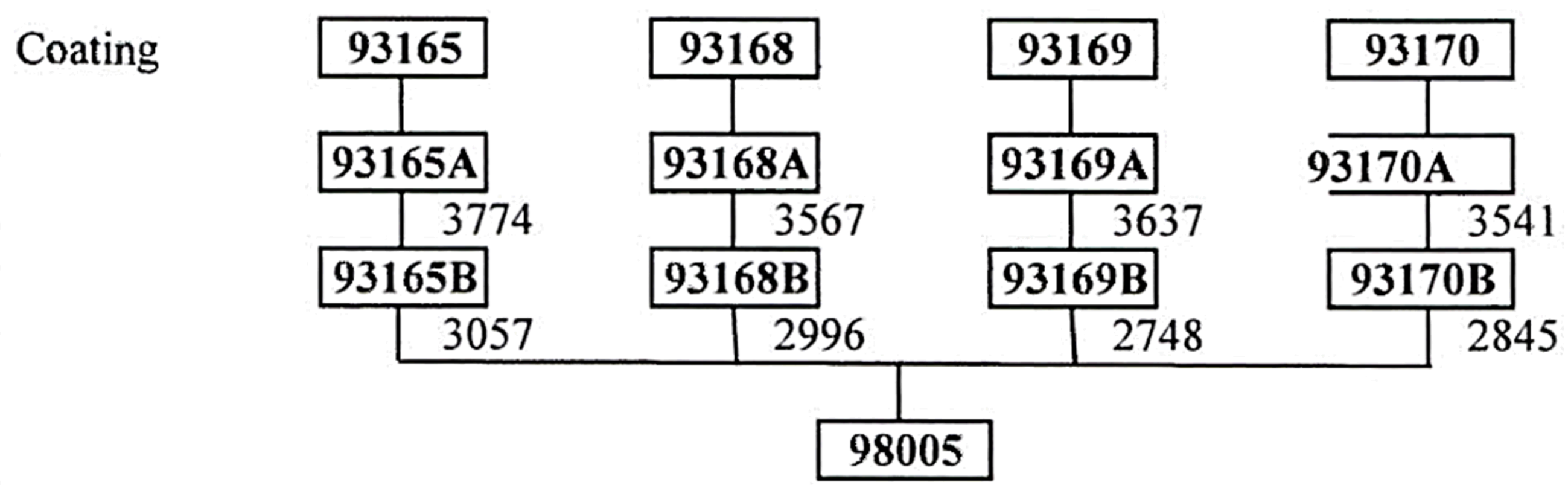




\section{Historical Overview - Compacts}

- TRISO particles are overcoated with a resin-infused graphite powder and pressed into cylindrical fuel "compacts"

- AGR-1, AGR-2, and AGR-3/4 fuels formed in laboratoryscale equipment

- Small tilted drum overcoater

- Pressed one at a time with weighed charges

" Two furnaces for carbonization and thermal treatment

- AGR-5/6/7 fuel formed in engineering-scale equipment

- 1-2 kg of TRISO overcoated in an hour in pharmaceutical equipment

- 4-hole automated press

" Single furnace for carbonization and thermal treatment

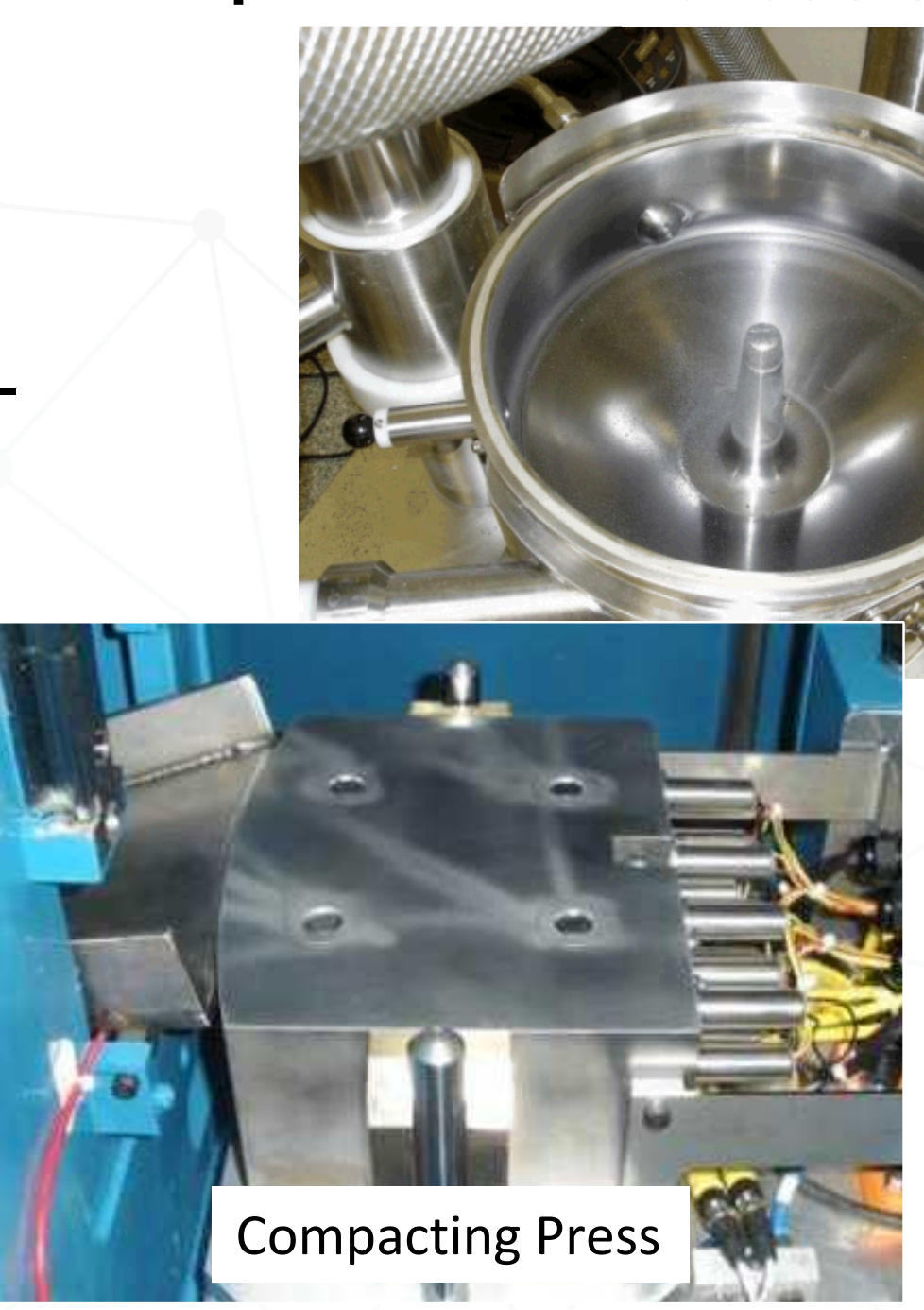




\section{Kernel Lot Characterization}

\begin{tabular}{|l|c|c|c|}
\hline \multicolumn{1}{|c|}{ J52R-16-69317 } & Specification & Mean & Status* \\
\hline Diameter $(\mu \mathrm{m})$ & $425 \pm 10$ & 425.8 & Pass \\
\hline Envelope density $\left(\mathrm{g} / \mathrm{cm}^{3}\right)$ & $\geq 10.4$ & 11.05 & Pass \\
\hline Uranium fraction $(\mathrm{gU} / \mathrm{gUCO})$ & $\geq 0.885$ & 0.897 & Pass \\
\hline 235U enrichment $\left(\mathrm{g}^{235} \mathrm{U} / \mathrm{gU}\right)$ & $0.155 \pm 0.001$ & 0.1548 & Pass \\
\hline $\mathrm{C}: \mathrm{U}$ (atomic ratio) & $0.40 \pm 0.10$ & 0.37 & Pass \\
\hline $\mathrm{O}: \mathrm{U}$ (atomic ratio) & $1.50 \pm 0.20$ & 1.441 & Pass \\
\hline (C+O)/U (atomic ratio) & $\leq 2.0$ & 1.811 & Pass \\
\hline $\begin{array}{l}\text { Individual impurities }(\mathrm{ppmw}): \\
\text { Li, Na, Al, Cl, } \mathrm{Ca}, \mathrm{V}, \mathrm{Cr}, \mathrm{Mn}, \mathrm{Fe}, \mathrm{Co}, \mathrm{Ni}, \mathrm{Cu}, \text { and } \mathrm{Zn}\end{array}$ & $\leq 100$ each & $5-25$ & Pass \\
\hline Process impurities (ppmw): P, S & $\leq 1,500$ each & $(\mathrm{P}) 5$ & Pass \\
\hline Aspect ratio & $\leq 1.05 @ .95 / .90$ & 1.012 & Pass \\
\hline
\end{tabular}

* Pass $\equiv$ meets all specifications on the mean and dispersion critical limits. 


\section{Kernel Lot Composition and Characterization}
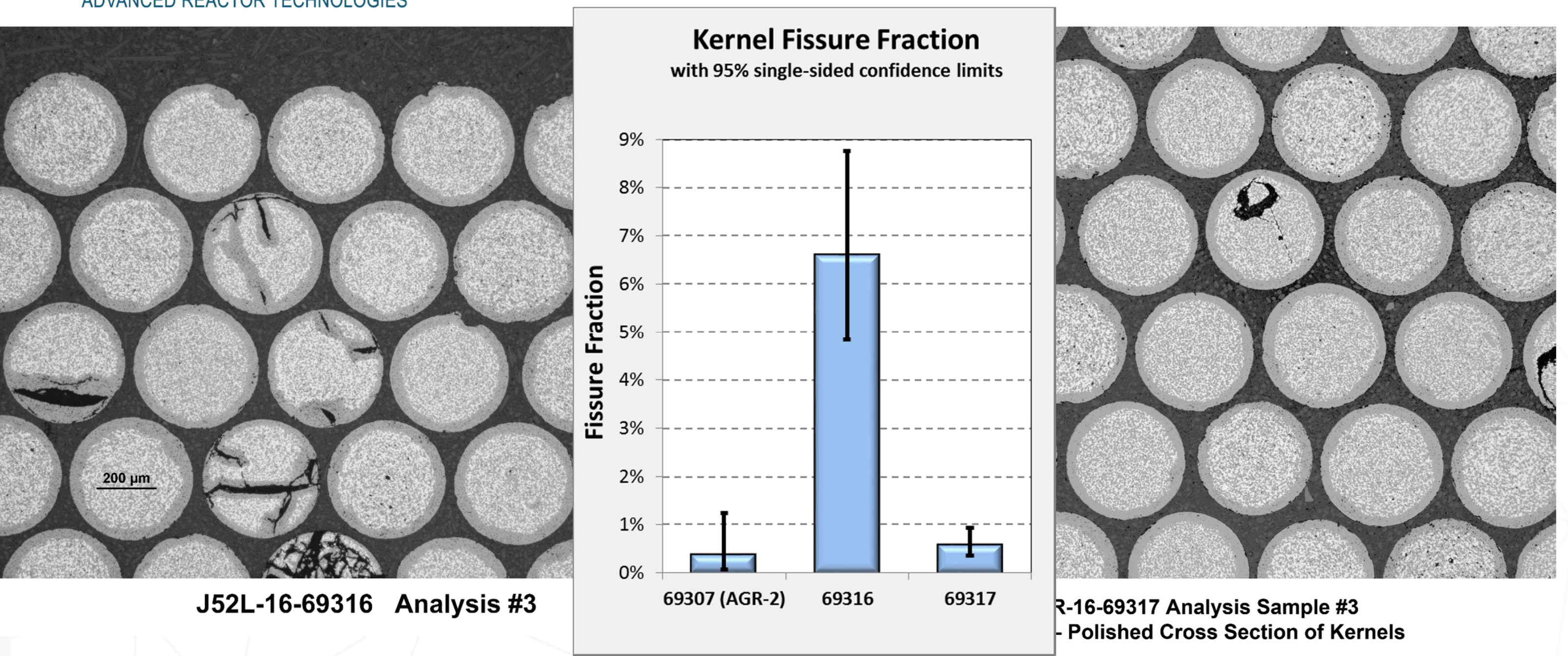


\section{TRISO Lot Composition and Characterization}

\begin{tabular}{|c|c|c|c|}
\hline J52R-16-98005 & Specification & Mean & Status $^{*}$ \\
\hline Buffer thickness $(\mu \mathrm{m})$ & $100 \pm 15$ & 100.4 & Pass \\
\hline IPyC thickness $(\mu \mathrm{m})$ & $40 \pm 4$ & 39.24 & Pass \\
\hline SiC thickness $(\mu \mathrm{m})$ & $35 \pm 3$ & 36.15 & Pass \\
\hline OPyC thickness $(\mu \mathrm{m})$ & $40 \pm 4$ & 35.03 & Low mean \\
\hline Buffer density $\left(\mathrm{g} / \mathrm{cm}^{3}\right)$ & $1.05 \pm 0.10$ & 1.031 & Pass \\
\hline IPyC density $\left(\mathrm{g} / \mathrm{cm}^{3}\right)$ & $1.90 \pm 0.05$ & 1.897 & Pass \\
\hline SiC density $\left(\mathrm{g} / \mathrm{cm}^{3}\right)$ & $\geq 3.19$ & 3.195 & Pass \\
\hline OPyC density $\left(\mathrm{g} / \mathrm{cm}^{3}\right)$ & $1.90 \pm 0.05$ & 1.897 & Pass \\
\hline
\end{tabular}

* Pass $\equiv$ meets all specifications on the mean and dispersion critical limits. 


\section{TRISO Lot Composition and Characterization}

\begin{tabular}{|l|c|c|c|}
\multicolumn{1}{|c|}{ J52R-16-98005 } & Specification & Mean & Status* \\
\hline IPyC diattenuation & $\leq 0.0170$ & 0.0153 & Pass \\
\hline OPyC diattenuation & $\leq 0.0122$ & 0.0102 & Pass \\
\hline SiC aspect ratio (faceting) & $\leq 1.14 @ .95 / .99$ & 1.053 & Pass \\
\hline Defective IPyC coating fraction & $\leq 1.0 \times 10^{-4}$ & $4.5 \mathrm{E}-5$ & Pass \\
\hline Defective OPyC defect fraction & $\leq 3.0 \times 10^{-4}$ & $0 / 35 \mathrm{k}$ & Pass \\
\hline Dispersed U Fraction & $(\leq 1.0 \mathrm{E}-5)^{\star *}$ & $1.04 \mathrm{E}-5$ & High \\
\hline Exposed Kernel Fraction & $(\leq 5.0 \mathrm{E}-5)^{* *}$ & $9.40 \mathrm{E}-6$ & Acceptable \\
\hline SiC Defect Fraction & $(\leq 1.0 \mathrm{E}-4)^{* *}$ & $1.89 \mathrm{E}-5$ & Acceptable \\
\hline
\end{tabular}

* Pass $\equiv$ meets all specifications on the mean and dispersion critical limits.

** Compact specification isn't relevant to TRISO particles. 


\section{Compact Characterization}

- Four batches of overcoated TRISO were separately pressed and divided across four furnace runs for thermal treatment

\begin{tabular}{|c|c|c|c|c|}
\hline $\begin{array}{c}\text { Pressing } \downarrow \text { Furnace } \\
\rightarrow\end{array}$ & A & B & C & D \\
\hline $\mathbf{1 4 1 5 4}(39.4 \%$ PF $)$ & 108 & 108 & 108 & 140 \\
\hline $\mathbf{1 4 1 5 5}(39.3 \%$ PF $)$ & 108 & 108 & 108 & 160 \\
\hline $\mathbf{1 4 1 5 6}(26.1 \%$ PF $)$ & 108 & 108 & 108 & 36 \\
\hline $\mathbf{1 4 1 5 7}(25.9 \%$ PF $)$ & 108 & 108 & 108 & 0 \\
\hline
\end{tabular}

- A-series compacts were used in AGR-5/6/7 while the $C$ and D-series were used for destructive characterization

- Pressure sensor failure interrupted furnace run B between carbonization and heat-treatment cycles. The other three runs used a continuous or "combined" cycle from carbonization through thermal treatment 


\section{Compact Characterization}

\begin{tabular}{|l|c|c|c|c|c|}
\multicolumn{1}{|c|}{ Property } & Specification & $14154 \mathrm{~A}$ & $14155 \mathrm{~A}$ & $14156 \mathrm{~A}$ & $14157 \mathrm{~A}$ \\
\hline $\begin{array}{l}\text { Mean U loading } \\
\text { (gU/compact) }\end{array}$ & & & & \\
$\quad \begin{array}{l}\text { Nominally 40\% PF } \\
\text { Nominally 25\% PF }\end{array}$ & $1.36 \pm 0.10$ & 1.428 & 1.388 & --- & --- \\
\hline Diameter $(\mathrm{mm})$ & $12.20 \leq \mathrm{D} \leq 12.44$ & 12.293 & 12.291 & 12.237 & 12.260 \\
\hline Length $(\mathrm{mm})$ & $24.40 \leq \mathrm{L} \leq 25.30$ & 25.094 & 24.692 & 24.996 & 24.752 \\
\hline Matrix density $\left(\mathrm{g} / \mathrm{cm}^{3}\right)$ & $\geq 1.65$ & 1.73 & 1.73 & 1.76 & 1.74 \\
\hline
\end{tabular}




\section{Compact Characterization}

\begin{tabular}{|c|c|c|c|c|c|}
\hline Property & Spec & $14154 C$ & 14155C & $14156 C$ & $14157 C$ \\
\hline Iron $(\mu \mathrm{g})$ & $\leq 25$ & \multicolumn{4}{|c|}{$<5$} \\
\hline $\begin{array}{l}\text { Transition metals }(\mu \mathrm{g}) \\
\mathrm{Cr}, \mathrm{Mn}, \mathrm{Co}, \text { and } \mathrm{Ni}\end{array}$ & $\leq 50$ each & \multicolumn{4}{|c|}{$\mathrm{Cr}<25$, all others $<10$} \\
\hline Calcium $(\mu \mathrm{g})$ & $\leq 50$ & \multicolumn{4}{|c|}{$<25$} \\
\hline Aluminum & $\leq 50$ & \multicolumn{4}{|c|}{$<25$} \\
\hline Titanium + Vanadium & $\leq 240$ & \multicolumn{4}{|c|}{$<20$} \\
\hline
\end{tabular}




\section{BWXT-NOG Compact Characterization}

\begin{tabular}{|c|c|c|c|}
\hline & & 40\% PF Compacts & 25\% PF Compacts \\
\hline Property & Spec & $14154 C$ and $14155 C$ & $14156 C$ and $14157 C$ \\
\hline Defective OPyC & $\leq 0.01$ & 0/4200 (7.1E-4 @ 95\%) & 0/4200 (7.1E-4 @ 95\%) \\
\hline Dispersed U Fraction & $\leq 1 \mathrm{E}-5$ & 3.18E-5 (3.80E-5 @ 95\%) & 2.66E-5 (2.95E-5 @ 95\%) \\
\hline Exposed Kernel Fraction & $\leq 5 \mathrm{E}-5$ & 6.57E-5 (9.28E-5 @ 95\%) & 7.39E-6 (1.48E-5 @ 95\%) \\
\hline SiC Defect Fraction & $\leq 1 \mathrm{E}-4$ & 6.96E-5 (9.66E-5 @ 95\%) & 9.24E-5 (1.22E-4 @ 95\%) \\
\hline
\end{tabular}

Unexpected results:

The DUF increased to $3 x$ the TRISO lot

The SDF increased $4 x-5 x$

Inconsistencies in analytical outcomes made some results suspect 


\section{Compact Characterization}

- Confirmatory analysis was requested by ORNL

" J52R-16-11034 overcoated TRISO (40\% PF)

- 40 compacts from J52R-16-14154C (40\% PF) compacts

- 29 compacts from J52R-16-14156C and 11 compacts from J52R-16-14156D (25\% PF)

- ORNL observed unusual analytical results

- Deconsolidate is a thick slurry and difficult to clarify the supernate

- The populations analyzed by ORNL are insufficient to reject any data

- Additional analyses are needed to improve statistics 


\section{Compact Characterization}
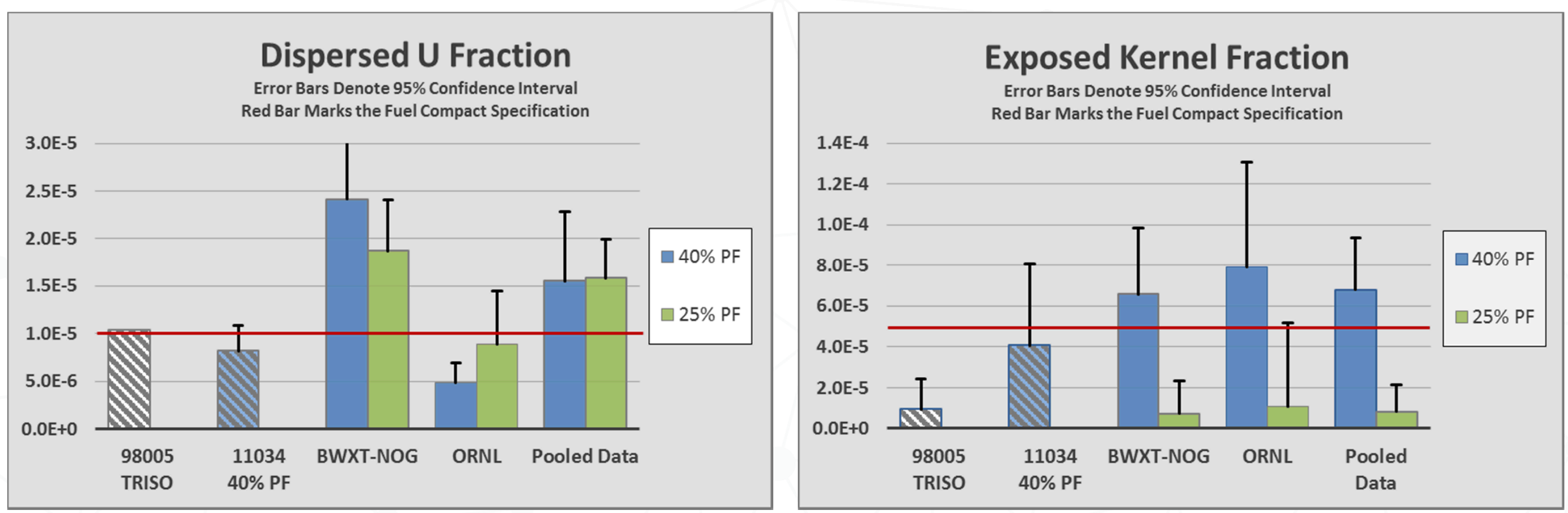


\section{Compact Characterization}

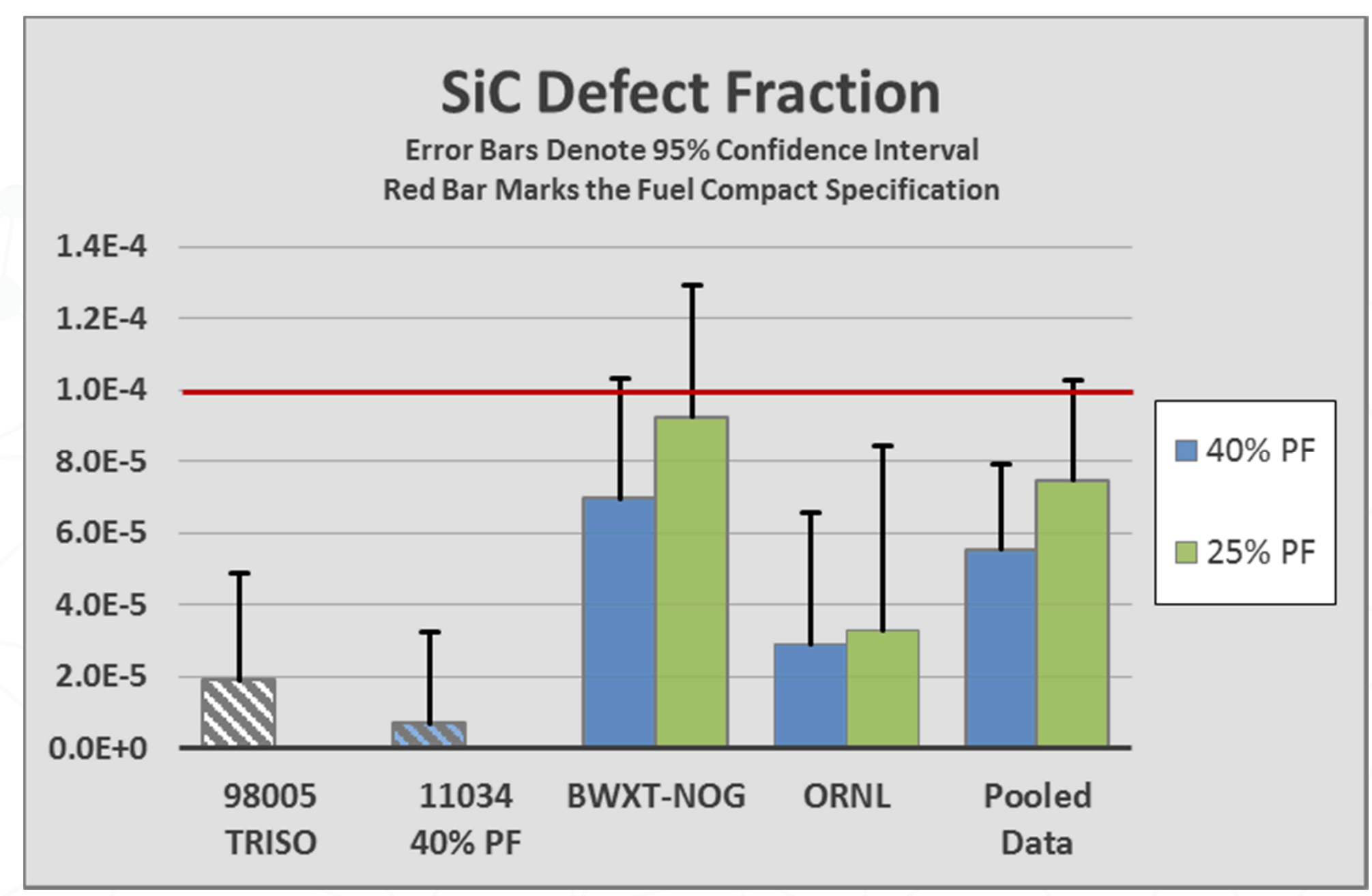




\section{Summary}

- Kernels

- $17 \mathrm{~kg}$ of LEUCO kernels were certified

- All product specifications were met

- Fissure fraction is comparable to AGR-2 UCO fuel

- TRISO Particles

- $11.1 \mathrm{~kg}$ of LEUCO TRISO particles were certified

- All product specifications were met except for OPyC thickness

- Mean DUF was high, but EKF and SDF were $\sim 1 / 5^{\text {th }}$ of the compact specification

- Fuel Compacts

- Fuel compacts of both packing fractions meet all specifications except for DLBL defects

- DUF is above the specification for both PFs

- EKF for $25 \%$ PF compacts meet specification

- EKF for $40 \%$ PF compacts fail the specification; mostly from damage in the overcoater

- SDF for both PFs should meet specs with more data

- ORNL should be tasked to analyze more samples of all compact batches 


\section{ART}

\section{Douglas Marshall}

\section{TRISO Fuel Fabrication Technical Lead}

Douglas.Marshall@inl.gov

(208) 526-3657

ART.INL.gov 


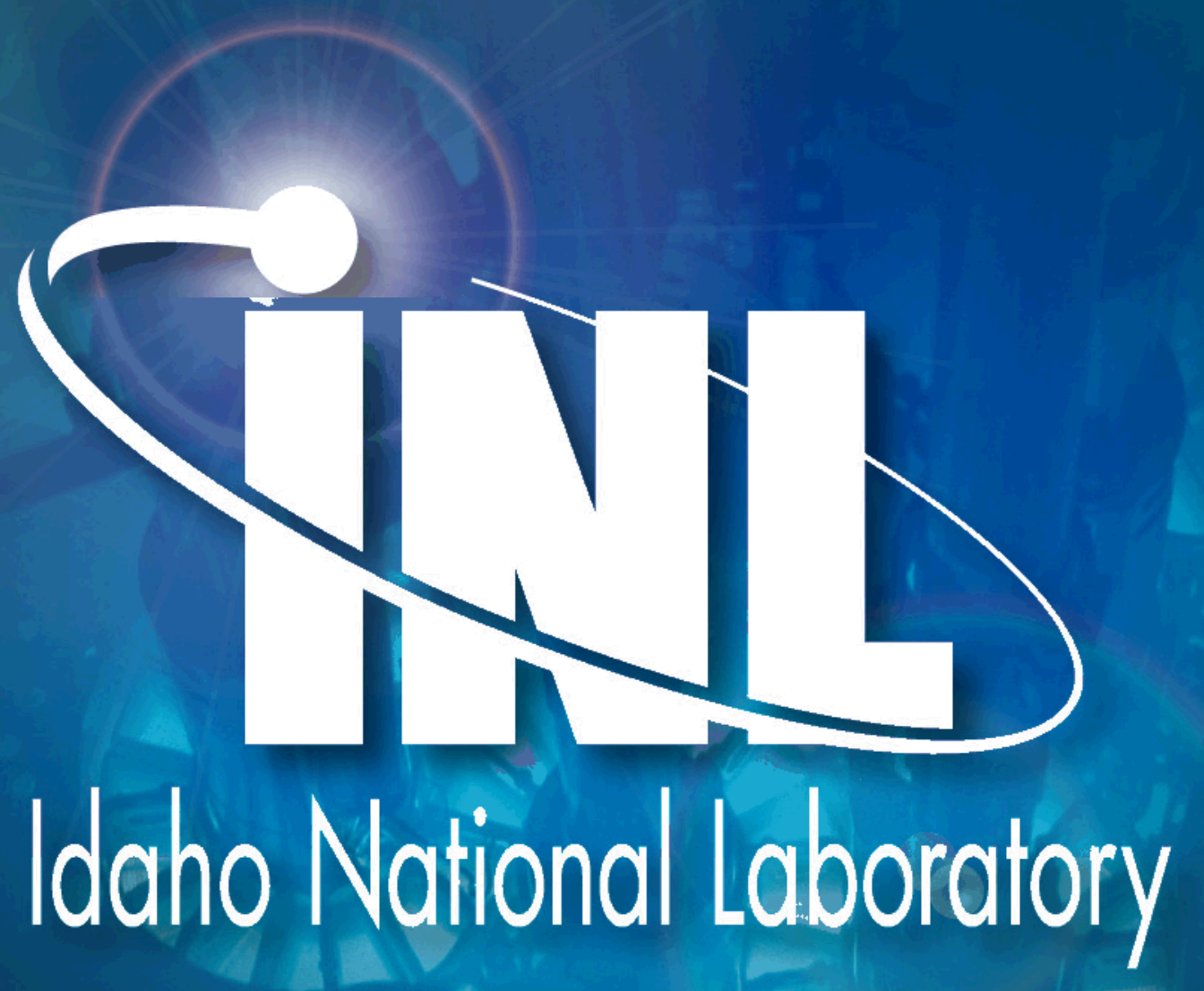




\section{Compact Characterization}

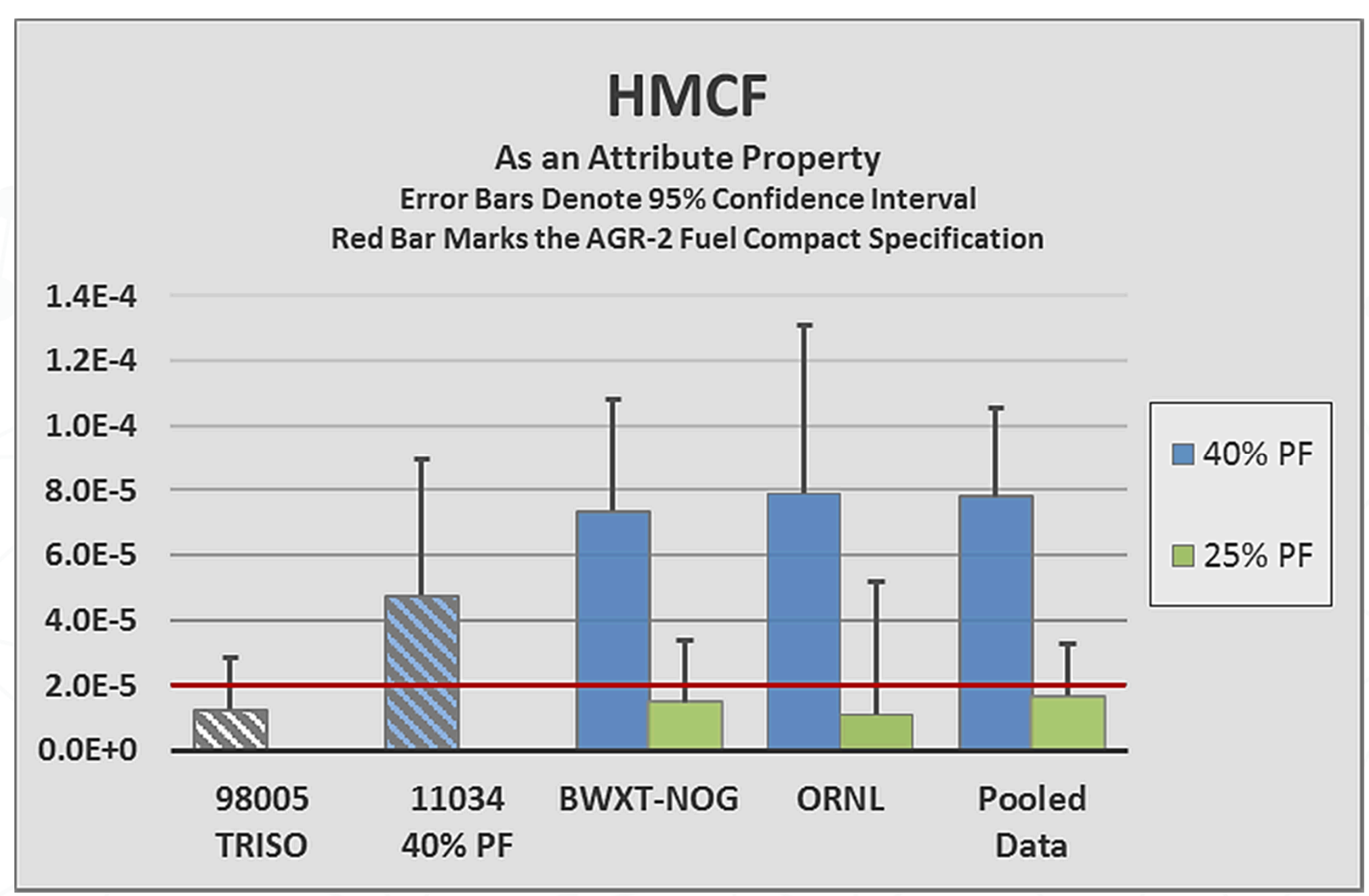

